6. Thewissen, J. G. M. \& Madar, S. J. Syst. Biol. 48, 21-30 (1999). 7. Luckett, W. P. \& Hong, N. J. Mamm. Evol. 5, 127-182 (1998). 8. Graur, D. \& Higgins, D. Mol. Biol. Evol. 11, 357-364 (1994).

9. Gatesy, J., Hayashi, C., Cronin, M. \& Arcander, P. Mol. Biol. Evol. 13, 954-963 (1996).

10. Gatesy, J., O’Grady, P. \& Baker, R. H. Cladistics 15, 271-313 (1999).

11. Ursing, B. M. \& Arnason, U. Proc. R. Soc. Lond. B 265, 2251-2255 (1998).
12. Nikaido, M., Rooney, A. \& Okada, N. Proc. Natl Acad. Sci. USA 99, 10261-10266 (1999).

13. Hillis, D. M. Proc. Natl Acad. Sci. USA 96, 9979-9981 (1999).

14. Miyamoto, M. M. Curr. Biol. 9, R816-R819 (1999).

15. Bajpai, S. \& Gingerich, P. D. Proc. Natl Acad. Sci. USA 95 15464-15468 (1998).

16. Kumar, S. \& Hedges, S. B. Nature 392, 917-920 (1997).

Photonuclear physics

\title{
Laser light splits atom
}

\author{
Donald Umstadter
}

asers have become ubiquitous, being used in everything from a bar-code reader to a compact disk player. Who would have thought that they might be used to split the atom? A few scientists proposed to do just that more than a decade ago ${ }^{1}$. But accomplishing it in the laboratory had to await the maturity of new technology ${ }^{2}$, which enabled the construction of the world's most powerful lasers at the Lawrence Livermore National Laboratory in the United States and at the Rutherford Appleton Laboratory in the United Kingdom. Now two independent research teams have used these lasers to split the uranium atom ${ }^{3,4}$. This work, reported in Physical Review Letters, is just the latest milestone in the race to discover what happens when matter interacts with the highest electromagnetic field strengths of light.

Over the past century, the peak power of artificial light sources has increased exponentially, from a kilowatt $\left(10^{3}\right.$ watts $)$ to a petawatt ( $10^{15}$ watts). This corresponds to an increase of more than a factor of ten each decade (Fig. 1). These dramatic advances have led to new physical insights and applications along the way. For instance, it was the invention of the cathode ray tube, used to make X-rays at the turn of the last century (and still used to this day for most television displays), that led to the discovery of electrons. Later, the invention of the laser increased the power of light to the point that it could accelerate an electron to close to the speed it naturally has as it orbits the nucleus of the atom. Remarkably, the distance required to reach this speed is but a millionth of a metre, the light's own wavelength. Having laser fields almost comparable to atomic fields has led to the discipline of physics known as nonlinear optics.

With the latest increase in laser power, electrons can now be accelerated to even higher speeds, close to the speed of light itself, again in a distance of just the laser wavelength. At such high speeds, electrons are governed by the laws of special relativity, leading to new types of nonlinear motion ${ }^{5}$. Gamma rays (energetic X-rays) are emitted when the highly energetic 'relativistic' elec-

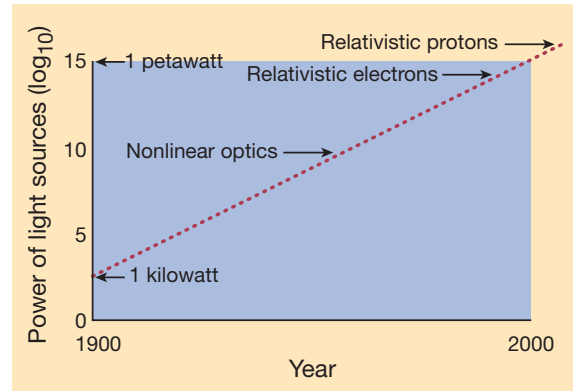

Figure 1 As the power of laboratory light sources increases, the energy of accelerated particles increases accordingly, making laser-induced nuclear reactions possible. The most powerful lasers have now reached petawatt $\left(10^{15}\right.$ watts) peak powers, allowing them to accelerate electrons to relativistic speeds. The gamma rays produced by such electrons have been used in recent experiments to split the uranium atom ${ }^{3,4}$.

trons accelerate rapidly during a collision with neighbouring ions. If these gamma rays are energetic enough, they can then perturb the delicate stability of heavy nuclei such as uranium, causing it to split into lighter elements and neutrons in a process called photonuclear fission. This is exactly what the two groups of laser scientists have just demonstrated.

The fission of uranium usually brings to mind nuclear reactors. But before you get your hopes up that splitting atoms with lasers will solve the world's energy problems, it should be pointed out that more energy is required to power the laser than is released through the fission process. Besides, there are much more efficient ways to accelerate electrons and produce gamma rays than by using optical light - for instance, with accelerators driven by radiofrequency waves.

Because photofission has been studied with radio-frequency accelerators since they were invented decades ago, it is now quite well understood. In fact, a simple search of a database of physics journals for articles published since 1967 using the keyword "photofission" yields a total of 463 hits. Only seven of these appeared in the journal Physical Review Letters. Usually, the number of articles published in this exclusive physics journal on a particular topic tapers off as our fundamental knowledge of the subject increases. So why were references 1, 3 and 4 the only ones in the past 15 years to enjoy this privilege? As the authors readily admit, it seems less to do with what they tell us about photofission than with the tantalizing prospect that lasers can now be used to induce nuclear processes and the myriad applications that may follow.

The most familiar laser-driven nuclear process is fusion (the inverse of fission), in which lasers are used to heat hydrogen nuclei to temperatures greater than that of the Sun in order to fuse them together to make helium and release energy. Indeed, one of the main reasons for the current interest in the fission papers ${ }^{3,4}$ is that the relativistic electrons produced by these experiments might some day be used as a spark plug to ignite a fusion reaction.

Another laser-driven nuclear reaction that might soon be possible is the creation of an ionized gas (plasma) of positrons as well as electrons. (Positrons are anti-electrons.) High-density antimatter is difficult to produce on Earth because it annihilates upon encountering ordinary matter, but it is of interest to astrophysicists because it might exist elsewhere in the Universe, for example in pulsars, quasars and black holes. Remarkably, with compact powerful lasers, dense electron-positron plasmas might soon be available for study in university laboratories.

Several groups have used electrons accelerated by high-power lasers to accelerate ions to high velocities ${ }^{6,7}$. The ions can be used to produce high concentrations of short-lived radioactive isotopes to tag cancer cells in nuclear medicine. More energetic protons from larger and more expensive radiofrequency-driven accelerators are even being used to kill cancer cells.

As the power of lasers continues to increase, the day will come when protons can even be accelerated to relativistic velocities and, possibly, directly by the laser field itself. (Protons are more difficult to accelerate than electrons because they are thousands of times more massive.) This next era of laser-matter interactions will involve all sorts of other nuclear reactions that could lead to novel applications, some of which are anyone's guess.

Donald Umstadter is at the Center for Ultrafast Optical Science and the Department of Nuclear Engineering and Radiological Science, University of Michigan, Ann Arbor, Michigan 48109, USA. e-mail:dpu@umich.edu

1. Boyer, K., Luk, T. S. \& Rhodes, C. K. Phys. Rev. Lett. 60, 557-559 (1988).

2. Strickland, D. \& Mourou, G. Opt. Commun. 56, 219-221 (1985). 3. Ledingham, K. W. D. et al. Phys. Rev. Lett. 84, 899-902 (2000).

4. Cowan, T. E. et al. Phys. Rev. Lett. 84, 903-906 (2000).

5. Chen, S.-Y., Maksimchuk, A. \& Umstadter, D. Nature 396, 653-655 (1998).

6. Sarkisov, G. et al. Phys. Rev. E 59, 7042-7054 (1999). 7. Krushelnick, K. et al. Phys. Rev. Lett. 83, 737-740 (1999). 